\title{
A Ligation of the Lacrimal Excretory Duct in Mouse Induces Lacrimal Gland Inflammation with Proliferative Cells
}

\author{
Ying Liu, ${ }^{1}$ Masatoshi Hirayama, ${ }^{1,2}$ Tetsuya Kawakita,, ${ }^{1,3}$ and Kazuo Tsubota ${ }^{1}$ \\ ${ }^{1}$ Department of Ophthalmology, Keio University School of Medicine, Shinjuku, Japan \\ ${ }^{2}$ Regulatory Biology Laboratory, Salk Institute for Biological Studies, La Jolla, CA, USA \\ ${ }^{3}$ Department of Ophthalmology, Kitasato Institute Hospital, Kitasato University, Minato, Japan \\ Correspondence should be addressed to Tetsuya Kawakita; kawakita@a2.keio.jp
}

Received 28 November 2016; Revised 12 May 2017; Accepted 21 May 2017; Published 10 August 2017

Academic Editor: Hung-Fat Tse

Copyright (c) 2017 Ying Liu et al. This is an open access article distributed under the Creative Commons Attribution License, which permits unrestricted use, distribution, and reproduction in any medium, provided the original work is properly cited.

\begin{abstract}
The lacrimal gland secretes tear fluids to ocular surface, which plays an indispensable role in maintaining the health of the ocular epithelia and protecting the ocular surface from the external environment. The dysfunction of the lacrimal glands causes dry eye disease due to a reduction in tear volume. The dry eye disease is becoming a popular public disease, for the number of patients is increasing, who have subjective symptom and loss of vision, which affect the quality of life. Inflammatory change in the damaged lacrimal gland has been reported; however, a major challenge is to establish a simple animal model to observe the changes. Here, we demonstrated an injury model by ligating the main excretory duct of the lacrimal gland, which is a simple and stable way to clearly understand the mechanism of lacrimal gland inflammation. We observed the process of injury and proliferation of the lacrimal gland and detected a population of lacrimal gland epithelial cells with proliferation potential which were also nestin-positive cells following duct ligation. This study successfully established an injury model to observe the tissue injury process of the lacrimal gland, and this model will be useful for analysis of the inflammation and proliferation mechanism in the future.
\end{abstract}

\section{Introduction}

The lacrimal gland (LG) is a secretory gland that secretes tear fluids to maintain the health of the ocular surface epithelium and protect the ocular surface from the external environment [1]. Dysfunction of the LG leads to dry eye disease (DED), an ocular surface disease characterized by insufficient tear production and damaged epithelium [2]. A significance to treat DED based on the understanding on mechanism on pathological changes in the LG has increased because DED has become one of common eye diseases in elderly people that causes subjective symptoms including discomfort as well as objective signs such as foreign body sensation, ocular irritation, and blurred vision, which affect the quality of life [3-5].

The LG is composed of various kinds of cells such as acinar, duct, and myoepithelial cells. The acini express water channel proteins including aquaporin 5 (AQP5) for aqueous tear secretion [6]. The myoepithelial cells that surround the acini help to secrete tear contents from acini in response to nerve stimulation [6]. To analyze the mechanism of tear decreasing after injury to the LG, several animal models, which could induce inflammation of the LG, have been proposed [7]. The single injection of IL-1, a cytokine, to the LG induced the inflammation partially and temporarily, and it caused reversible change after 7 days [8]. The reports indicated a regeneration capacity in the partial damaged LG [9]. An establishment of animal models for an efficient LG injury has been required to elucidate pathological changes against persistent injuries to the LG [10-12].

Duct ligation of organs has been a conventional and promising method to induce organ dysfunctions including inflammation and fibrosis by obstructing the fluid pathway, blood vessels in the bile duct, and liver arteries [13, 14]. 
Obstruction of the fluid pathway itself has recently been recognized as a novel pathology to lead meibomian gland dysfunctions [15-17]. However, it has been still controversial that the duct ligation of the lacrimal gland excretory duct can cause lacrimal gland inflammation efficiently in an animal model.

Here, we demonstrated a mouse model to induce LG injury by the ligation of the lacrimal gland excretory duct. The duct ligation caused stable and throughout injury of the LG; we observed the process of injury and proliferation of the LG and detected a population of LG epithelial cells with proliferation potential, which was also a population of cells positive for nestin, the stem/progenitor cell marker. Our current study provides evidence of a LG injury pattern by duct ligation, and this model will be useful for analysis of the mechanism and process of lacrimal gland inflammation and identification of a candidate stem/progenitor cell of the LG in the future.

\section{Methods}

2.1. Animals. Thirty-nine male C57BL/6Jc1 WT mice (7-8 weeks old) were purchased from CLEA Japan Inc. Mice were feed in a controlled environment following the guidelines of Keio University. The care and handling of the animals were performed in accordance with the NIH guidelines. All of the experimental protocols were approved by the Animal Care and Use Committee of the Keio University (Approval number 09167).

2.2. Duct Ligation of the Main Lacrimal Gland Excretory Duct. We established a mouse injury model by ligating the main excretory duct of the LG; this procedure induced injury to the tissue of the LG, which is shown in Figure 1(b). Animals were anesthetized using $2 \mathrm{mg} / \mathrm{mL}$ ketamine (Lot: V29D; Ciba Specialty Chemicals Holding Inc.) and $2 \mathrm{mg} / \mathrm{mL}$ xylazine (Lot: KP035HU, Bayer Holding Ltd.) mixed in saline $(10 \mu \mathrm{L} / \mathrm{g})$ by intraperitoneal injection. A small incision (less than $8 \mathrm{~mm}$ ) was made to the right side face skin. We gently lifted up the LG to expose the main excretory duct and then ligated the duct together with the vessel using a silicon tube $(0.2 \times 0.3 \mathrm{~mm}, 1-8194-02$ No.2, Justis, Japan). After the operation, the skin underwent two interrupted sutures with 10-0 nylon (Lot: 03-9315, Alcon). The left LG was not operated on and served as a control. Mice in the sham group had the right side skin cut but did not undergo LG ligation. The animals were sacrificed 3, 7, or 14 days after ligation. LGs were removed immediately after sacrifice.

2.3. Measurement of Tear Secretion. A phenol redimpregnated cotton thread was used to measure the aqueous tear production of experimental animals without anesthesia. The cotton thread was placed on the inner canthus gently without touching the ocular surface for $30 \mathrm{~s}$, and the length of the part which was stained red was measured as previously reported [18]. We measured aqueous tear production before operation and $3,7,9,12$, and 14 days after surgery.
2.4. Sample Collection and Weight Measurement. All LG specimens were removed from the surrounding tissue entirely, washed in PBS, dried, and weighed immediately with an electronic balance. The LG specimens were then cut in half to be used for real-time PCR, and the other half was fixed in OCT, immediately frozen in liquid nitrogen, and stored at $-80^{\circ} \mathrm{C}$ until stained.

2.5. Histological Analysis of the LG. LG specimens were cut into sections $(5-6 \mu \mathrm{m})$ for $\mathrm{H} \& \mathrm{E}$ and immunohistochemical staining. For $\mathrm{H} \& \mathrm{E}$ staining, the tissue sections were fixed with $10 \%$ formalin for 10 min and then stained with hematoxylin and $1 \%$ eosin. For immunohistochemical staining, the fresh tissue sections $(5 \mu \mathrm{m})$ were fixed with $4 \%$ paraformaldehyde (PFA; Wako, Osaka, Japan) in PBS and then blocked with 10\% normal donkey/goat serum (IHR-8135, ImmunoBioScience Corp., USA; S-1000, Lot: Y0322, Vector Laboratories, CA, USA) with $0.01 \%$ Triton X-100 (3550115, Nacalai) in PBS. After blocking, the tissue sections were incubated with the appropriate primary antibody overnight. The next day, after washing with PBS, the sections were incubated with the secondary antibody (1/300) and $0.1 \%$ Hoechst (1/1000) for 1 hour and observed. The following are the primary antibodies used for immunostaining: rabbit cytokeratin 14 (K14, 1/1000; PRB-155p, Covance); mouse $\alpha$-smooth muscle actin ( $\alpha$-SMA, 1/500, ab18640, Abcam), which is a marker of myoepithelial cells; rabbit AQP5 (1/100, SC-28628, Santa Cruz); and nestin (1/80; AF2736, $\mathrm{R} \& \mathrm{D})$, which is a neural stem cell marker; to evaluate the proliferation of acinar and duct cells after ligation, LG specimens were incubated with PCNA (1/100; M0879, Dako) as a cell proliferation marker. Anti-PCNA antibody was performed by a DAB staining kit (Lot: 415172, Nichirei Biosciences Inc.). A TUNEL assay was performed using the ApopTag Red apoptosis detection kit (Millipore, Bedford, MA, USA), according to the manufacturer's protocol. Histological sections were observed under the fluorescence microscope (BIOREVO BZ-9000) and Imager (Axiocam; Carl Zeiss Inc.). Imaging of the whole dish was captured and processed using BIOREVO (Keyence Corporation, Osaka, Japan), and the nestin-positive and CD45-positive area was calculated as pixel numbers.

2.6. RNA Extraction and Real-Time PCR. Total RNA was extracted from cells using TRIzol reagent (Invitrogen, Carlsbad, CA, USA) following the manufacturer's protocol. A NanoDrop 1000 Spectrophotometer (Thermo Scientific) was used to measure RNA concentration. Synthesis of cDNA from total RNA was performed using ReverTra Ace ${ }^{\circledR}$ qPCR RT Master Mix (FSQ-201, Toyobo, Japan). Quantitative real-time PCR was performed using the StepOnePlus equipment (Applied Biosystems) with TaqMan ${ }^{\circledR}$ Fast Universal PCR Master Mix (2X), without AmpErase ${ }^{\circledR}$ UNG (Applied Biosystems), and predesigned primers for cytokeratin 14, $\alpha$-SMA, AQP5, nestin, and glyceraldehyde-3-phosphate dehydrogenase $(\mathrm{GAPDH})$. The $\triangle \triangle \mathrm{CT}$ value calculated by StepOne Software (v2.2.2) was used to evaluate the mRNA levels and was normalized to GAPDH mRNA. 

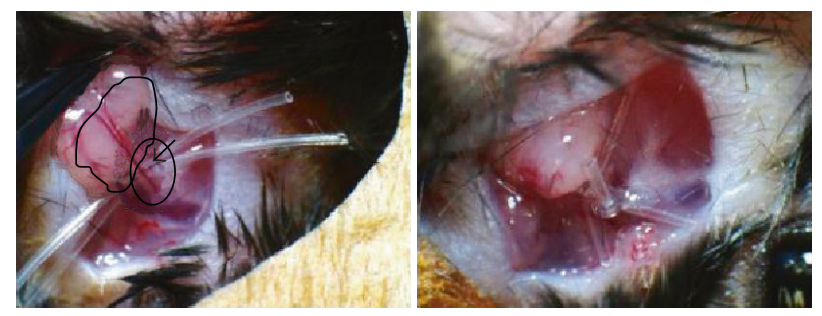

(a)
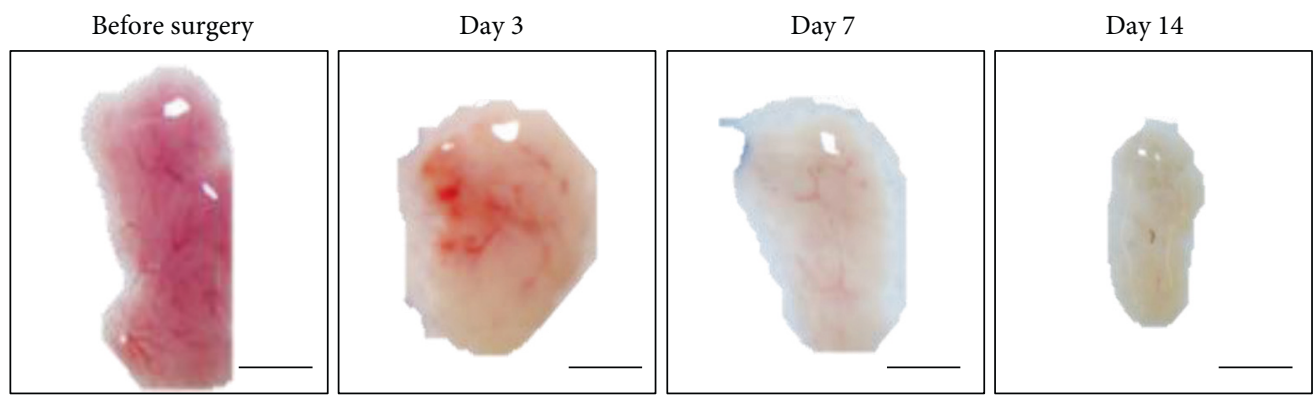

(b)

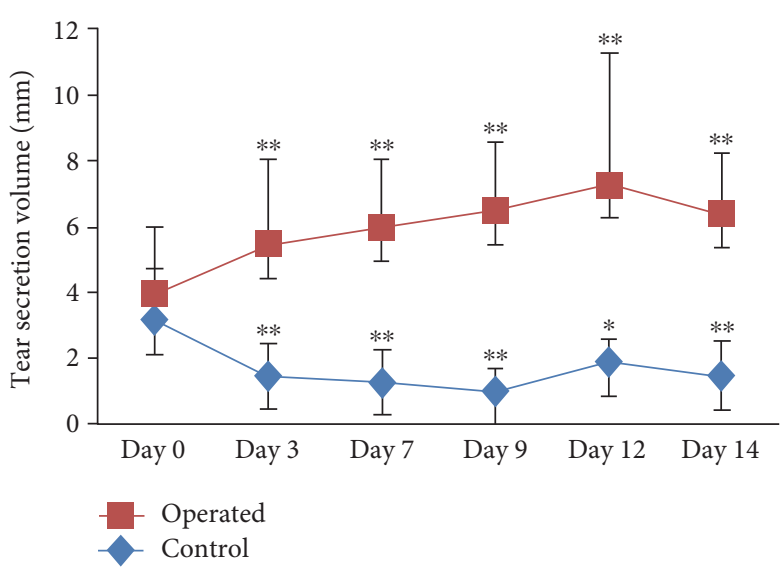

(c)

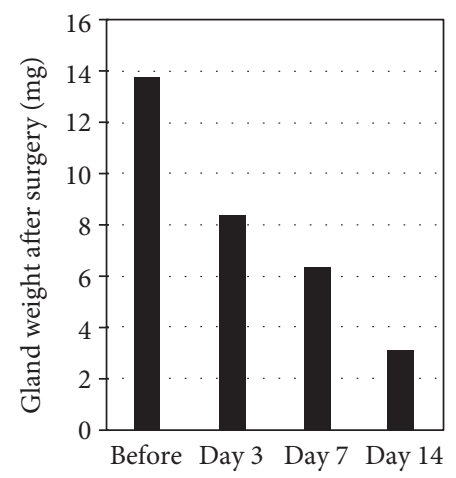

(d)

FIgure 1: The lacrimal duct ligation in mouse. (a) The photos of the lacrimal duct ligation procedure in mice. The white circle showed the excretory duct of the LG and the black circle showed the main LG. The procedure before ligation (left) and after ligation (right) was shown. (b) The macroscopic changes in the LG before and after ligation. The scale bar is $2 \mathrm{~mm}$. (c) The change in the average of tear secretion volume before and after ligation. Each group has more than 20 samples. ${ }^{*} p<0.05,{ }^{* *} p<0.01$. (d) The change in the gland weight before and after surgery. Each group has more than 20 samples.

\section{Results}

3.1. Macroscopic Changes in the Injured LG by the Duct Ligation. We first demonstrated the ligation of the mouse lacrimal excretory duct to investigate whether the procedure could cause LG injury (Figure 1(a)). After the ligation procedure, we analyzed the macroscopic changes in the LG before surgery and at days 3, 7, and 14 after surgery. The macroscopic observation showed that at 3, 7, and 14 days postligation, the LG was diminished and hardened and the transparency decreased (Figure 1(b)). To confirm the ligation effect of the duct, we also analyzed the change in tear secretion volume before and after surgery. We performed the ligation procedure on the right eyes, and we used the left eye as the control group without duct ligation (sham surgery group). In the right eye with duct ligation, tear secretion at 3 ,
$7,9,12$, and 14 days after surgery was significantly decreased compared with that in the eye in the sham surgery group (Figure 1(c)). Interestingly, the control showed a significant increase compared with day 0 at each time point due to compensation. We next analyzed the weight change during the procedure. The weight of the LG was decreased after surgery during the procedure (Figure $1(\mathrm{~d})$ ). These results indicated that the ligation of the lacrimal excretory duct was a success and could induce throughout injury in the LG.

3.2. Histological Changes in the LG after the Duct Ligation. We next investigated the pathological changes in the ductligated LG by histological analysis. We observed histological changes in the LG before surgery and following 3, 7, and 14 days after ligation by H\&E staining. Before surgery, the $H \& E$ staining showed the normal secretory gland histology 

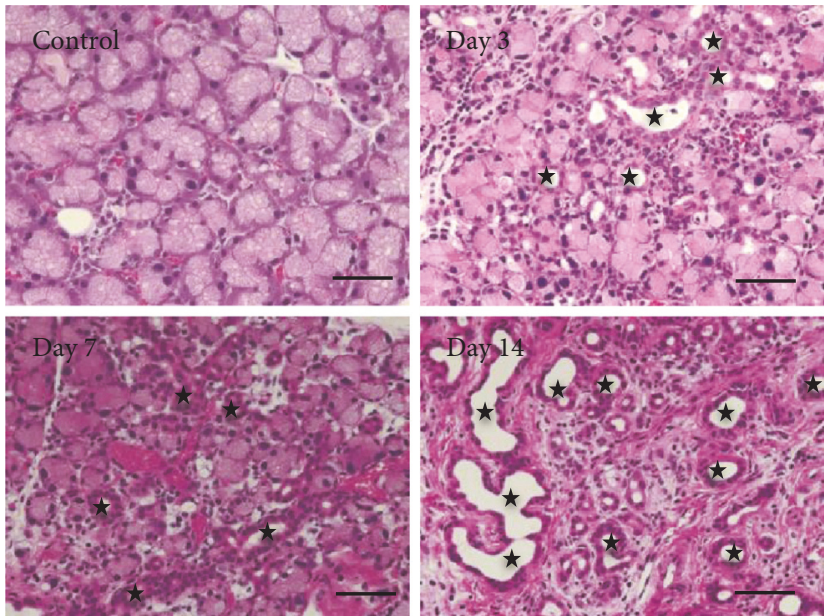

(a)

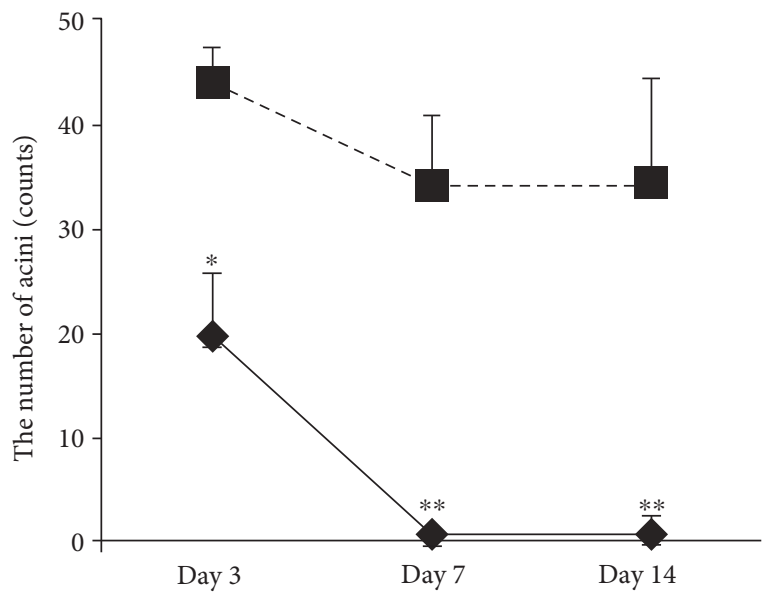

(b)

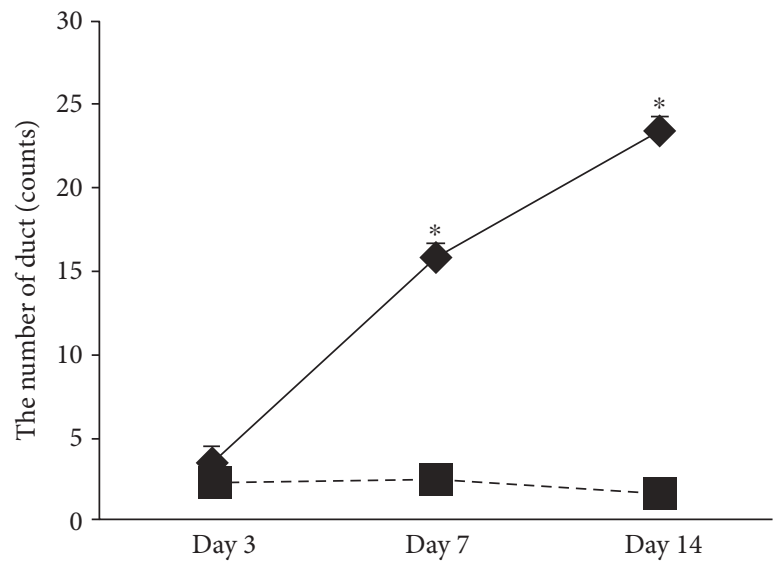

(c)

Figure 2: Histological changes in the LG after the ligation. (a) The representative images of the H\&E staining of the LG before and after surgery. $\star$ showed the enlarged and generated duct cavities. The scale bar is $50 \mu \mathrm{m}$. (b) The change in the average number of acini after surgery. ${ }^{*} p<0.05,{ }^{* *} p<0.01$. (c) The change in the average number of duct after surgery. ${ }^{*} p<0.05$.

including acini and ducts. After surgery, the results showed the infiltration of inflammatory cells into the connective tissue and around the duct area, especially at day 3 postligation. Extensive injury was observed at day 3 , and the peak of the injury was observed at day 7. After 14-day surgery, almost all the acini were diminished, with only few remaining; on the other hand, many residual and newly formed ducts were observed (Figure 2(a)). Quantitative analysis of acini and duct that unite following ligation also showed that the number of acini was significantly decreased and the number of ducts was significantly increased (Figures 2(b) and 2(c)). These results indicated that the LG after the duct ligation induced inflammation and atrophic changes, whereas some proliferative changes such as a duct increase occurred.

3.3. mRNA Expression Changes in the LG after the Duct Ligation. To clarify the proliferative changes in inflammatory status in the LG after the duct ligation, we performed realtime PCR analysis to observe gene expression changes in the LG by a time course. We analyzed the relative mRNA expression changes about cytokeratin 14 (KRT14) that was expressed in duct and myoepithelial cells, aquaporin 5 (AQP5) that was expressed in acinar lumens, and $\alpha$ SMA that was expressed in myoepithelial cells as representative markers in duct, acinar, and myoepithelial cells (Figure 3(a)). The expression of KRT14 and $\alpha$-SMA in the LG with duct ligation significantly increased temporarily with a peak at day 3. On the other hand, the expression of AQP5 was significantly decreased after the surgery. We next investigated the expression of nestin, a stem/progenitor marker, in the LG after duct ligation. The relative expression level of nestin also increased with a peak at day 7 (Figure 3(b)). These results indicated that the LG after the duct ligation induced not only atrophic inflammation but also proliferation of cells. The cells expressing KRT14 and $\alpha$-SMA proliferated immediately after injury, while cells expressing nestin increased following that.

3.4. Immunohistological Analysis on Inflammation and Proliferative Changes in the Duct-Ligated LG. To clarify the detail of the effect of duct ligation on the LG, we have analyzed inflammation and proliferative status in the LG 

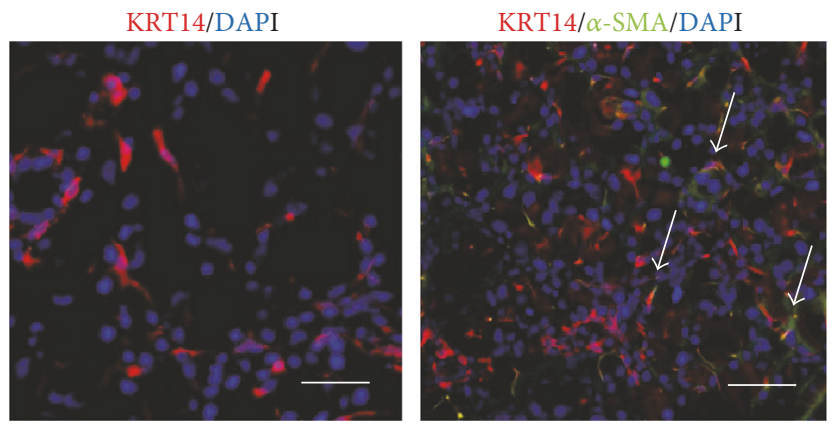

(a)

$\alpha$-SMA

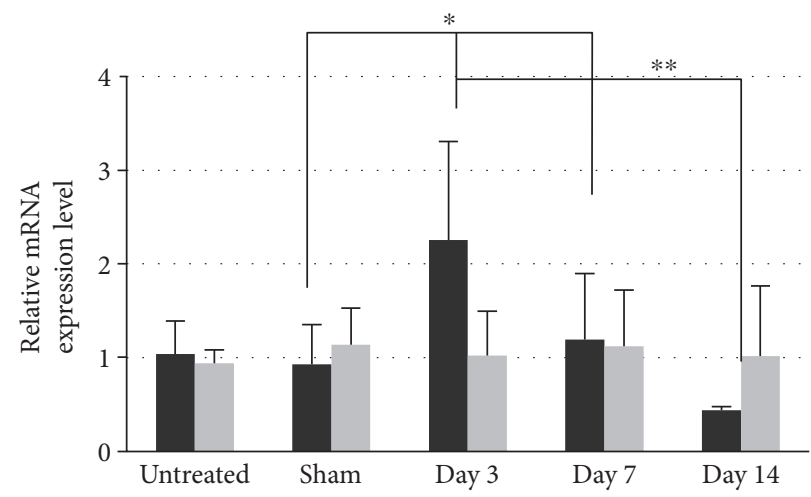

AQP5

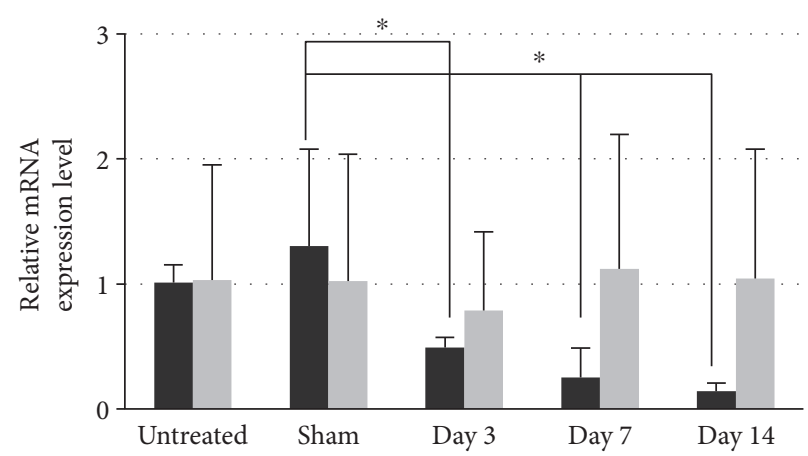

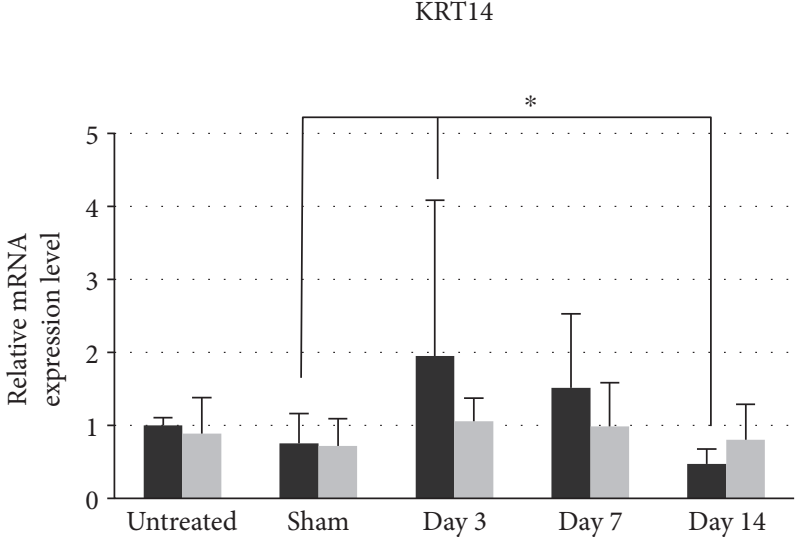

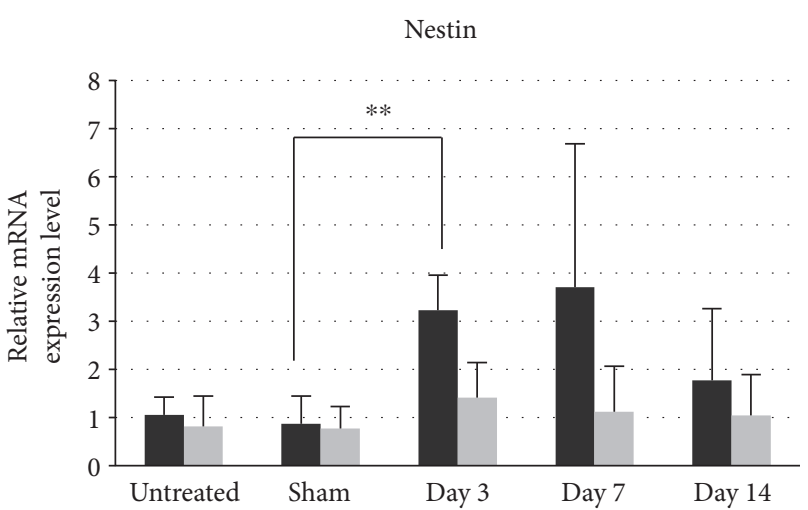

(b)

FIGURE 3: Expression changes in representative markers in the LG after surgery. (a) The histoimmunochemical staining of KRT14 and $\alpha$-SMA in the normal LG (red showed the KRT14, green showed the $\alpha$-SMA, and arrow showed the cells with both expression). The scale bar is $50 \mu \mathrm{m}$. (b) The difference of relative mRNA expression level of the representative markers before and after surgery. ${ }^{*} p<0.05$ and ${ }^{* *} p<0.01$.

after the procedure by immunohistological analysis. First, we examined the inflammation condition at each time point using CD45, a lymphocyte common antigen, which is expressed on all leukocytes. We observed an infiltration of large number of $\mathrm{CD} 45^{+}$cells at day 3 and day 7 , and it gradually diminished at day 14 following the ligation (Figure 4(a)). We next evaluated cell apoptosis induced by ligation by TUNEL staining. A few TUNEL-positive cells were found in acini and ducts at day 3, and TUNELpositive cells increased in the luminal layer of the ducts at day 7 , converging in one area, which was near the ligation site, and duct cell in the basal layer survived. TUNEL was almost negative at day 14 after surgery (Figure 4(b)). These suggested that the peak of inflammation occurred in the early stage and the inflammation decreased gradually.

To evaluate the proliferative process in the LG after the duct ligation, we analyzed the expression of PCNA, which was expressed in proliferative cells. The PCNA-positive $\left(\mathrm{PCNA}^{+}\right)$cells were found in the basal layer of the stratified duct epithelium above the basement membrane at day 3 and also in interstitial tissues. At day 7 , most $\mathrm{PCNA}^{+}$cells were found in acini and only a few were in the basal layer of the ducts. At day 14, the $\mathrm{PCNA}^{+}$cells were localized in small simple duct epithelium (Figures 4(c) and 4(d)). These 
CD45/DAPI
Before

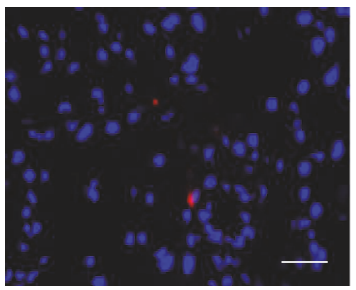

TUNEL staining

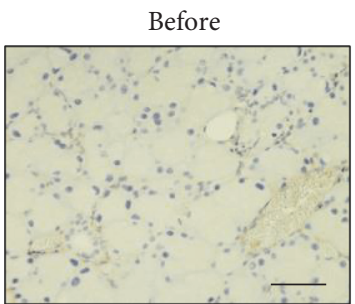

PCNA/DAPI

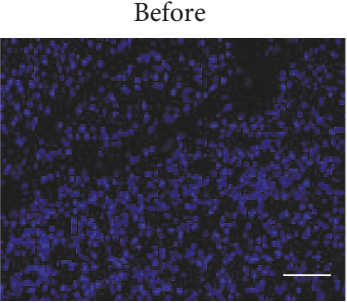

Day 3

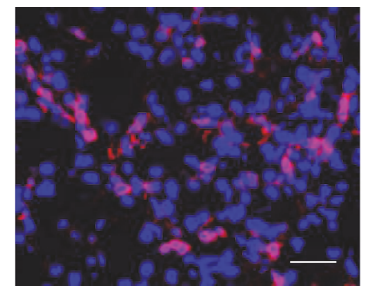

Day 7

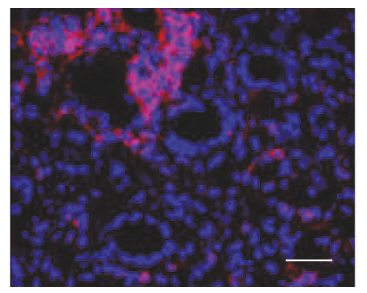

(a)
Day 14

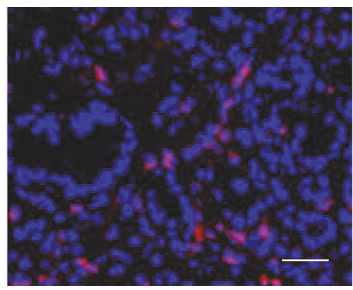


Nestin/DAPI
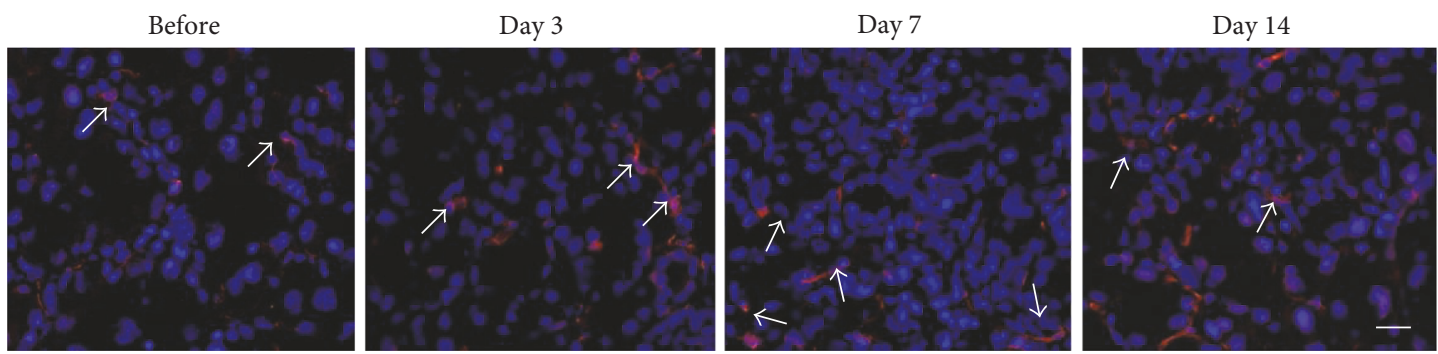

(a)

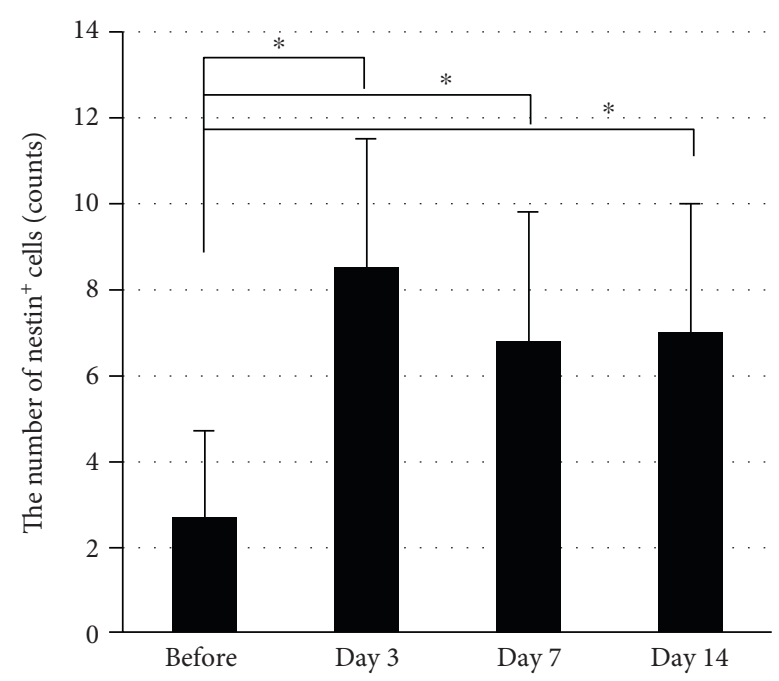

(b)

FIGURE 5: The increase in nestin-positive cells in the LG after surgery. (a) The time-course observation of cells with nestin expression before and after surgery (red and arrows showed the nestin). The scale bar is $50 \mu \mathrm{m}$. (b) The change in the average number of cells per visual field with nestin expression before and after surgery. More than 10 samples in each group were included. ${ }^{*} p<0.05$.

myoepithelial cells and $\alpha$-SMA that was expressed in myoepithelial cells in the LG, and they were not expressed in nestin ${ }^{+}$cells (data not shown). These results suggested that the proliferation process after inflammation in the LG was supported by proliferative cells such as $\mathrm{PCNA}^{+}$cells in the basal layer in duct and nestin ${ }^{+}$cells in interstitial tissues in the LG.

\section{Discussion}

In this study, we have demonstrated the ligation of the LG excretory duct to establish a tissue injury model of the adult mouse LG. Our model could successfully induce the atrophic changes throughout the LG after the surgery. The procedure easily created injury in the LG over a time course and showed the inflammation and proliferative process after surgery, which is almost consistent with the other LG injury model. Our study clarified the time course change in the lacrimal gland after the duct ligation and showed the induced inflammation at the early stage and proliferative changes with $\mathrm{PCNA}^{+}$cells and nestin ${ }^{+}$cells. This model will be useful for investigation on character in such proliferative cells expressing a stem cell marker in the LG.
Animal models for efficient tissue damage have been reported for investigation on mechanism of inflammation, regenerative potential of organs, and isolation of stem/ progenitor cells from the target organs [8, 25-29]. Previous studies have shown that a partial radiation to salivary glands in mice leads to proliferation of cells, which have an ability to form salispheres for cell injection therapy to restore the salivary gland functions [30]. In the LG, some animal models for tissue damage using cytokines and bone marrow transplantation have been established, and they have revealed mechanisms of induced inflammation and regeneration abilities in the LG after injury [7, 31, 32]. The duct ligation procedure is simple and stable to lead to tissue inflammation in the liver, bile ducts, and salivary glands [13, 33-35]. However, the details on the effect of the duct ligation on the mouse LG have been unclear because no studies about them have been reported. We have successfully demonstrated a new tissue injury model of the mouse LG by duct ligation in this study.

Mechanisms of tissue damage in this model were based on both tear flow obstruction and ischemic changes by the ligation of duct and an artery running along with the duct [34]. We could confirm them by a tear flow decrease after the ligation and white color change in the LG in macroscopic 
observation. Anatomically, the major artery for the LG flows from two directions, front (along with the duct) and back sides [36]. The artery along with the duct supplies approximately $60 \%$ of blood flow to the LG. In this model, because the rest of blood supply was maintained [36], the LG could not lead to necrosis immediately. Such a partial injury status is crucial to observe inflammation status and proliferation status after the injury [8]. Our model could lead to proliferative status after inflammation as consistent with the previous partial injury model in the LG.

Current studies on regenerative medicine have revealed that proliferating cells after injury have a possibility to reconstruct the damaged tissues [37]. In our study, the small duct structure increased after surgery, which indicated a part of regeneration capacity of the LG. In the PCNA labeling experiment, we found that the $\mathrm{PCNA}^{+}$cells were first detected in the basal layer of the ducts at day 3 , the $\mathrm{PCNA}^{+}$cells proliferated at day 7 , and then most $\mathrm{PCNA}^{+}$cells were found in the simple layer of the ducts at day 14 . The temporal increase in $\mathrm{PCNA}^{+}$cells at the basal layer of the duct suggested the contribution to the increase in the small ducts. Nestin, the intermediate filament protein, has been focused as a stem/ progenitor marker of the central nervous system and the hair follicle [24]. Studies using nestin-driven GFP (ND-GFP) transgenic mice have revealed the possibility of the nestin ${ }^{+}$ hair follicle stem cells as an autologous source of adult stem cells to repair damaged tissues including those of nerves and cardiac muscles [38-40]. In previous reports, nestin ${ }^{+}$ cells, which were located in interstitial tissues, play an essential role in the repair of the LG after injury. Nestin ${ }^{+}$cells have been recognized as multilineage progenitor cells and gland-derived stem cells $[22,23]$. In our model, the nestin ${ }^{+}$ cells were also located in the interstitial tissues between acini and ducts and they increased after the duct ligation successfully. Interesting finding would be provided by the analysis of pluripotency and regeneration capacity of the nestin ${ }^{+}$cells in the LG.

\section{Conclusion}

In summary, we demonstrated the ligation of the LG excretory duct to induce partial tissue injury in the mouse LG. The current model could successfully induce the inflammation and proliferation process with the increase in cells expressing a stem cell marker. This mouse model will be a useful tool to observe the inflammation process and analyze the origin and regeneration capacity of proliferative cells, such as nestin ${ }^{+}$cells, in the LG to reveal mechanism to restore tissue damages.

\section{Conflicts of Interest}

The authors have no financial interests.

\section{Authors' Contributions}

Ying Liu and Masatoshi Hirayama equally contributed to this work.

\section{References}

[1] S. Mishima, "Some physiological aspects of the precorneal tear film," Archives of Ophthalmology, vol. 73, pp. 233-241, 1965.

[2] K. Tsubota, Y. Satake, and J. Shimazaki, "Treatment of severe dry eye," Lancet, vol. 348, p. 123, 1996.

[3] M. Uchino, M. Dogru, Y. Uchino et al., "Japan Ministry of Health study on prevalence of dry eye disease among Japanese high school students," American Journal of Ophthalmology, vol. 146, pp. 925-929, 2008.

[4] M. Uchino, Y. Nishiwaki, T. Michikawa et al., "Prevalence and risk factors of dry eye disease in Japan: Koumi study," Ophthalmology, vol. 118, pp. 2361-2367, 2011.

[5] E. Goto, Y. Yagi, M. Kaido, Y. Matsumoto, K. Konomi, and K. Tsubota, "Improved functional visual acuity after punctal occlusion in dry eye patients," American Journal of Ophthalmology, vol. 135, pp. 704-705, 2003.

[6] J. E. Schechter, D. W. Warren, and A. K. Mircheff, "A lacrimal gland is a lacrimal gland, but rodent's and rabbit's are not human," The Ocular Surface, vol. 8, pp. 111-134, 2010.

[7] S. Yaguchi, Y. Ogawa, T. Kawakita, S. Shimmura, and K. Tsubota, "Tissue renin-angiotensin system in lacrimal gland fibrosis in a murine model of chronic graft-versus-host disease," Cornea, vol. 34, Supplement 11, pp. S142-S152, 2015.

[8] D. Zoukhri, E. Macari, and C. L. Kublin, "A single injection of interleukin-1 induces reversible aqueous-tear deficiency, lacrimal gland inflammation, and acinar and ductal cell proliferation," Experimental Eye Research, vol. 84, pp. 894904, 2007.

[9] D. Zoukhri, "Mechanisms involved in injury and repair of the murine lacrimal gland: role of programmed cell death and mesenchymal stem cells," The Ocular Surface, vol. 8, pp. 60-69, 2010.

[10] M. Hirayama, T. Kawakita, K. Tsubota, and S. Shimmura, "Challenges and strategies for regenerating the lacrimal gland,” The Ocular Surface, vol. 14, pp. 135-143, 2016.

[11] M. Hirayama, M. Oshima, and T. Tsuji, "Development and prospects of organ replacement regenerative therapy," Cornea, vol. 32, Supplement 1, pp. S13-S21, 2013.

[12] M. Hirayama, K. Tsubota, and T. Tsuji, "Bioengineered lacrimal gland organ regeneration in vivo," Journal of Functional Biomaterials, vol. 6, pp. 634-649, 2015.

[13] C. G. Tag, S. Sauer-Lehnen, S. Weiskirchen et al., "Bile duct ligation in mice: induction of inflammatory liver injury and fibrosis by obstructive cholestasis," Journal of Visualized Experiments, no. 96, article 52438, 2015.

[14] J. M. Sheen, L. T. Huang, C. S. Hsieh, C. C. Chen, J. Y. Wang, and Y. L. Tain, "Bile duct ligation in developing rats: temporal progression of liver, kidney, and brain damage," Journal of Pediatric Surgery, vol. 45, pp. 1650-1658, 2010.

[15] To KW, R. J. Hofmann, and F. A. Jakobiec, "Extensive squamous hyperplasia of the meibomian duct in acne rosacea," Archives of Ophthalmology, vol. 112, pp. 160-161, 1994.

[16] R. Arita, K. Itoh, S. Maeda et al., "Meibomian gland duct distortion in patients with perennial allergic conjunctivitis," Cornea, vol. 29, pp. 858-860, 2010.

[17] V. S. Patel, D. R. Meyer, and J. A. Carlson, "Intratarsal keratinous cysts of the meibomian gland (a sebaceous duct cyst): report of 2 cases," The American Journal of Dermatopathology, vol. 33, pp. 624-627, 2011. 
[18] T. Kojima, T. H. Wakamatsu, M. Dogru et al., “Age-related dysfunction of the lacrimal gland and oxidative stress: evidence from the $\mathrm{Cu}, \mathrm{Zn}$-superoxide dismutase-1 (Sod1) knockout mice," The American Journal of Pathology, vol. 180, pp. 1879-1896, 2012.

[19] A. Richter, N. Nissen, P. Mailander et al., "Mammary glandderived nestin-positive cell populations can be isolated from human male and female donors," Stem Cell Research \& Therapy, vol. 4, p. 78, 2013.

[20] E. Gorjup, S. Danner, N. Rotter et al., "Glandular tissue from human pancreas and salivary gland yields similar stem cell populations," European Journal of Cell Biology, vol. 88, pp. 409-421, 2009.

[21] Y. Amoh, L. Li, K. Katsuoka, S. Penman, and R. M. Hoffman, "Multipotent nestin-positive, keratin-negative hair-follicle bulge stem cells can form neurons," Proceedings of the National Academy of Sciences of the United States of America, vol. 102, pp. 5530-5534, 2005.

[22] S. Nagel, F. Rohr, C. Weber et al., "Multipotent nestin-positive stem cells reside in the stroma of human eccrine and apocrine sweat glands and can be propagated robustly in vitro," PLoS One, vol. 8, article e78365, 2013.

[23] M. Kawashima, T. Kawakita, Y. Maida et al., "Comparison of telomere length and association with progenitor cell markers in lacrimal gland between Sjogren syndrome and non-Sjogren syndrome dry eye patients," Molecular Vision, vol. 17, pp. 1397-1404, 2011.

[24] L. Li, J. Mignone, M. Yang et al., "Nestin expression in hair follicle sheath progenitor cells," Proceedings of the National Academy of Sciences of the United States of America, vol. 100, pp. 9958-9961, 2003.

[25] D. Zoukhri, A. Fix, J. Alroy, and C. L. Kublin, "Mechanisms of murine lacrimal gland repair after experimentally induced inflammation," Investigative Ophthalmology \& Visual Science, vol. 49, pp. 4399-4406, 2008.

[26] M. Hirayama, M. Ogawa, M. Oshima et al., "Functional lacrimal gland regeneration by transplantation of a bioengineered organ germ," Nature Communications, vol. 4, p. 2497, 2013.

[27] M. Hirayama, "Proof of a concept for bioengineered organ replacement to restore lacrimal gland function," Nippon Ganka Gakkai Zasshi, vol. 119, pp. 799-806, 2015.

[28] M. Hirayama, Y. Liu, T. Kawakita, S. Shimmura, and K. Tsubota, "Cytokeratin expression in mouse lacrimal gland germ epithelium," Experimental Eye Research, vol. 146, pp. 54-59, 2016.

[29] M. Hirayama, K. SBH, T. Kawakita et al., "Identification of transcription factors that promote the differentiation of human pluripotent stem cells into lacrimal gland epitheliumlike cells," NPJ Aging and Mechanisms of Disease, vol. 3, no. 1, p. 1, 2017.

[30] Y. Sumita, Y. Liu, S. Khalili et al., "Bone marrow-derived cells rescue salivary gland function in mice with head and neck irradiation," The International Journal of Biochemistry \& Cell Biology, vol. 43, pp. 80-87, 2011.

[31] S. You, C. L. Kublin, O. Avidan, D. Miyasaki, and D. Zoukhri, "Isolation and propagation of mesenchymal stem cells from the lacrimal gland," Investigative Ophthalmology \& Visual Science, vol. 52, pp. 2087-2094, 2011.
[32] A. Gromova, D. A. Voronov, M. Yoshida et al., "Lacrimal gland repair using progenitor cells," Stem Cells Translational Medicine, vol. 6, pp. 88-98, 2017.

[33] K. Okumura, K. Nakamura, Y. Hisatomi et al., "Salivary gland progenitor cells induced by duct ligation differentiate into hepatic and pancreatic lineages," Hepatology, vol. 38, pp. 104-113, 2003.

[34] D. Galili, O. Maller, and V. J. Brightman, "The effects of desalivation by duct ligation or salivary gland extirpation on taste preference in rats," Archives of Oral Biology, vol. 26, pp. 853-858, 1981.

[35] N. Emmelin, J. R. Garrett, and P. Ohlin, "Secretory activity and the myoepithelial cells of salivary glands after duct ligation in cats," Archives of Oral Biology, vol. 19, pp. 275-283, 1974.

[36] A. Ducasse, J. F. Delattre, J. B. Flament, and J. Hureau, "The arteries of the lacrimal gland," Anatomia Clinica, vol. 6, pp. 287-293, 1984.

[37] A. Atala, "Tissue engineering, stem cells and cloning: current concepts and changing trends," Expert Opinion on Biological Therapy, vol. 5, pp. 879-892, 2005.

[38] Y. Amoh, L. Li, R. Campillo et al., "Implanted hair follicle stem cells form Schwann cells that support repair of severed peripheral nerves," Proceedings of the National Academy of Sciences of the United States of America, vol. 102, pp. 1773417738, 2005.

[39] A. Yamazaki, M. Yashiro, S. Mii et al., "Isoproterenol directs hair follicle-associated pluripotent (HAP) stem cells to differentiate in vitro to cardiac muscle cells which can be induced to form beating heart-muscle tissue sheets," Cell Cycle, vol. 15, pp. 760-765, 2016.

[40] R. M. Hoffman, Multipotent Stem Cells of the Hair Follicle, J. M. Walker, Ed., Humana Press, Springer Science+ Business Media New York, 2016. 

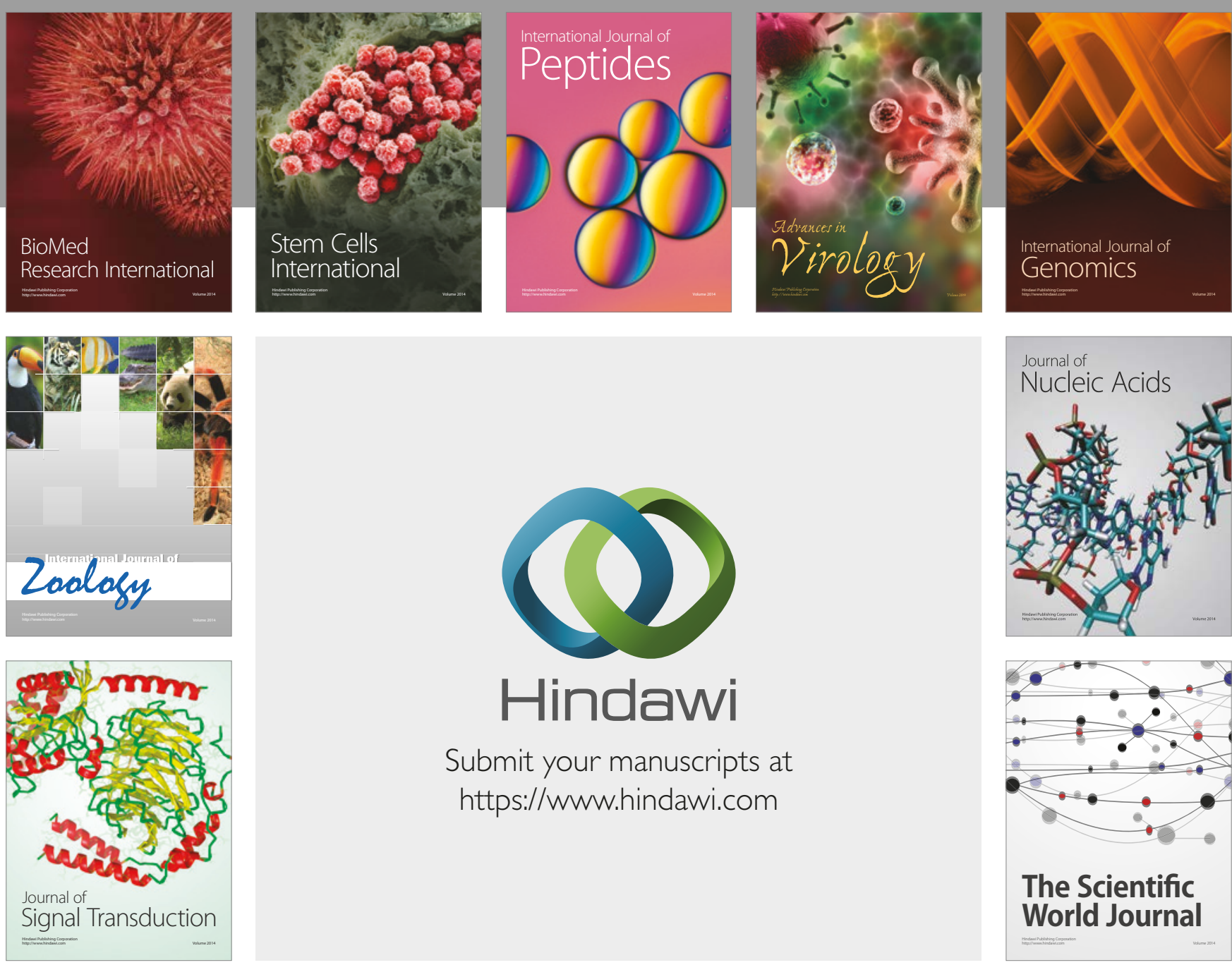

Submit your manuscripts at

https://www.hindawi.com
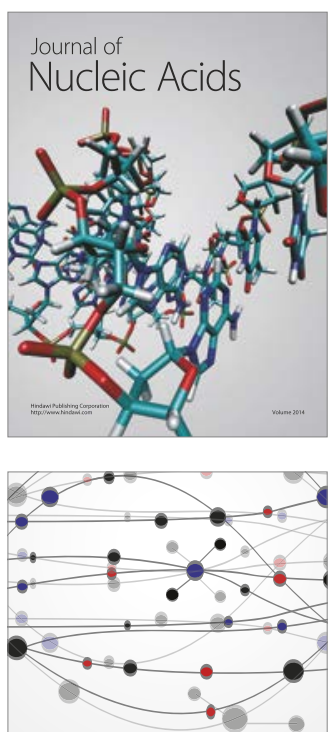

The Scientific World Journal

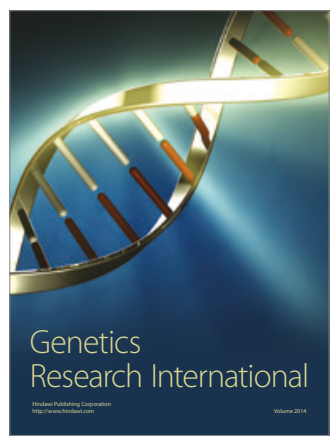

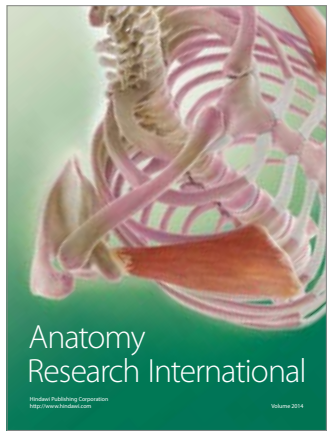

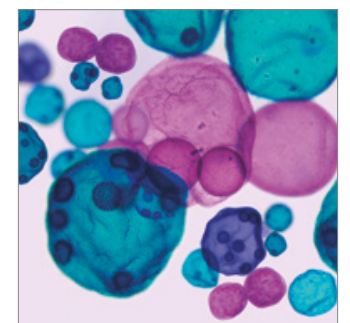

International Journal of Microbiology
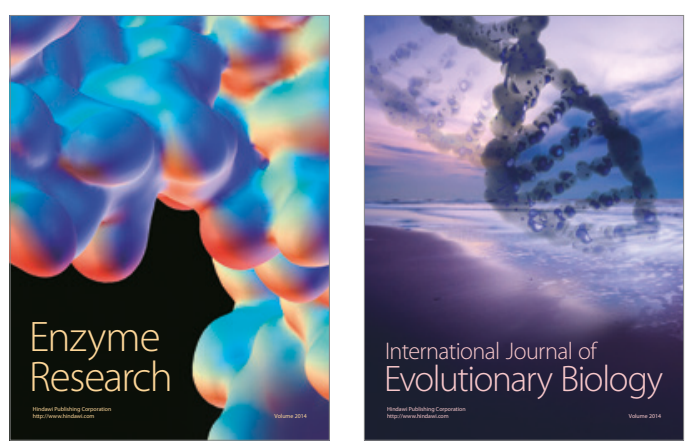
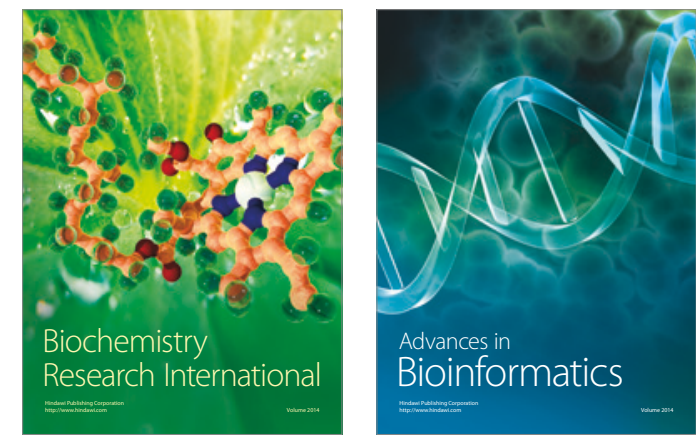

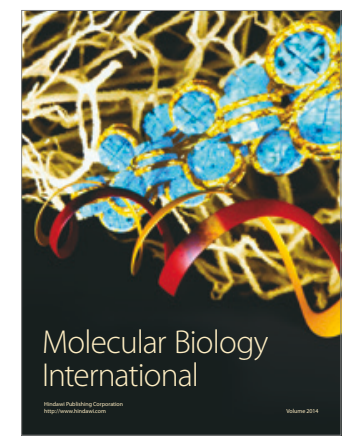

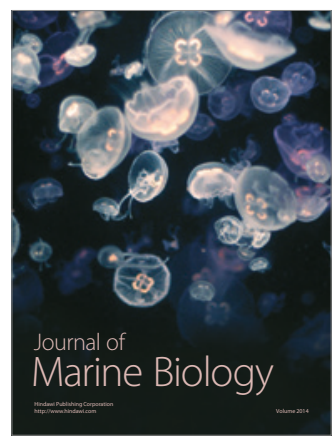

